\title{
Cold Chain Maintenance Evaluation of Pre- Cooked Pasta by Visible and Short Wave InfraRed Spectroscopy
}

\author{
Giuseppe Bonifazi \\ Department of Chemical \\ Engineering, Materials \& \\ Environment, \\ Sapienza University of Rome, \\ Rome, Italy \\ giuseppe.bonifazi@uniroma1.it
}

\author{
Giuseppe Capobianco \\ Department of Chemical \\ Engineering, Materials \& \\ Environment, \\ Sapienza University of Rome, \\ Rome, Italy \\ giuseppe.capobianco@uniroma1.it
}

\author{
Riccardo Gasbarrone \\ Department of Chemical \\ Engineering, Materials \& \\ Environment, \\ Sapienza University of Rome, \\ Rome, Italy \\ riccardo.gasbarrone@uniroma1.it
}

\author{
Silvia Serranti \\ Department of Chemical \\ Engineering, Materials \& \\ Environment, \\ Sapienza University of Rome, \\ Rome, Italy \\ silvia.serranti@uniroma1.it
}

\begin{abstract}
Pasta is widely used in many cuisines all around the world for its important nutritional properties. The quality assurance and the maintenance of the cold chain of pre-cooked pasta products have a significant impact in economic terms on the manufacturing companies. For this reason, a fast, reliable, not-destructive and non-invasive method is needed to fulfill the above-mentioned goals. Visible and Near InfraRed spectroscopy, coupled with chemometric analysis, are powerful tools that can make the production and supply of pre-cooked pasta more transparent, also reducing food waste. In this study, a spectrophotoradiometer operating in the Visible - Short Wave InfraRed (Vis-SWIR) range $(350-2500 \mathrm{~nm})$ was used to acquire reflectance spectra on pre-cooked pasta samples, with two levels of saltiness, produced in Italy and intended for the US market. Partial Least Squares - Discriminant Analysis (PLS-DA) classification models were calibrated and validated to recognize the samples according to their salting and physical conditions (i.e. frozen/thawed), starting from their spectral signatures. Classification performances showed promising ability in characterizing samples according to the previously mentioned attributes.
\end{abstract}

Keywords-Pre-cooked food, Visible and Near InfraRed Spectroscopy, multivariate analysis, cold chain maintenance, quality control applications.

\section{INTRODUCTION}

The world pasta market is constantly growing, according to consumer needs, in terms of ease of consumption and for pasta important nutritional properties. Pasta is one of the main and traditional Italian food products and is also very popular in many countries. Italy is the main producer (3.0 million tons) and consumer (about $28 \mathrm{~kg} /$ year per capita). In addition, Italy is also the main exporter of pasta (about 1.5 million tons). In recent years, the huge flow of exportations of pasta from Italy corresponds to about half of the national production [1]. In this scenario, it is necessary to ensure the quality of the product, which is one of the main key-issue to pursuit.
Dealing with frozen food and more specifically with reference to frozen pasta-based products, the set of chemicalphysical analyses that must be carried out in order to ensure the quality of the product and to control the cold chain maintenance has a significant economic impact on the manufacturing companies, furthermore, the results of these analyses are in many cases not immediately available.

In recent times, more and more companies working in the pasta sector are starting to use methods based on Near InfraRed (NIR) spectroscopy combined with typical methodologies of multivariate analysis (i.e. chemometrics), allowing quick investigations and the possibility to perform simultaneous determinations of different parameters [2-4].

Visible and NIR spectroscopy techniques are actually utilized to perform both qualitative and quantitative analysis in different fields: i.e. in primary/secondary raw materials sector $[5,6]$, in cultural heritage $[7,8]$, in the agricultural and food industry [9-14], in the pharmaceutical and chemical industry $[15,16]$, in clinical application [17-19] and more generally in analytical science [20] to perform systematic environmental remote and proximal sensing.

A spectrophotoradiometer operating in the Vis-SWIR range $(350-2500 \mathrm{~nm})$ was used to acquire reflectance spectra on precooked pasta samples, with two levels of saltiness, intended to be marketed in the United States of America. Spectra were acquired in frozen and thawed conditions.

Starting from the acquired spectra, Principal Component Analysis (PCA) was carried out to evaluate the differences between the two sets of samples (Mezze Penne and Pennette 72) at different levels of salting and according to their physical condition (i.e. frozen/thawed). Subsequently, Partial Least Squares - Discriminant Analysis (PLS-DA) classification models were calibrated and validated to recognize the samples according to their saltiness and to their physical condition. 


\section{MATERIALS AND METHODS}

\section{A. Sample preparation}

The pasta samples analyzed in this study were provided by a company Gelit SpA, located in Cisterna di Latina (Lazio, Italy). The analyzed samples consist of two types of precooked pasta, with two different degrees of salting: 6 samples of Mezze Penne and 6 samples of Pennette72. Approximately $200 \mathrm{~g}$ of each sample was placed in different plastic plates. Each sample was then analyzed in two stages: frozen (just taken out from the dry ice icebox) and thawed (after about two hours left to rest at a temperature of $26^{\circ} \mathrm{C}$ ), as shown in Fig. 1 .

\section{B. Reflectance spectra collection}

For each sample, 50 reflectance spectra were acquired (for a total of 600 spectra for each acquisition time) by an ASD FieldSpec $4{ }^{\circledR}$ Standard - Res spectrophotoradiometer equipped with a contact probe [21], in two different times: on frozen and thawed samples. The instrument was calibrated each time before acquiring a new sample set. Calibration was performed by referencing the dark current calibration file and by mean of the white reference measurement(i.e. standardized white Spectralon ${ }^{\circledR}$ ceramic material). Data acquisition and calibration procedure were carried out using the ASD RS3TM software.

\section{Data handling and preprocessing}

The spectra acquired with ASD RS3 ${ }^{\text {TM }}$ software in ".asd" format were exported to ASCII text files thanks to the ViewSpec Pro ${ }^{\text {TM }}$ software (ver. 6.2.0.). The files thus obtained were subsequently imported into the MATLAB ${ }^{\circledR}$ environment (MATLAB R2016b; ver. 9.1.0.) as a "dataset object" using a routine written precisely for the purpose: the "fieldspec_import2.m". The files imported into the MATLAB environment were then analyzed using the Eigenvector Research Inc. PLS toolbox (ver. 8.2.1) [22].

Spectral pre-processing algorithms were applied to data not only used to reduce instrument noise, scattering and other physical phenomena, but also to enhance sample differences. The first pre-processing algorithm applied to the spectral data was the so-called "splice correction" [23]. It was applied to eliminate the "gaps" located between the various acquisition domains of the three detectors of the ASD spectrometer. Subsequently, the acquired spectral data $(350-2500 \mathrm{~nm})$ were reduced to $450-2500 \mathrm{~nm}$. Several pre-processing strategies were thus explored. Finally, the pre-processing combination was chosen among those providing the best response in terms of data decomposition [24]. The sequential pre-processing chain thus was: Multiplicative Scatter Correction (MSC) and Mean Center (MC). MSC was used as a filter for the correction of scattering effects and $\mathrm{MC}$ to center data columns according to a zero.

\section{Exploratory analysis of the decomposed spectral data}

Principal component analysis was used to carry out an exploratory analysis of the spectral data, according to the type of sample and to the physical condition of the sample (frozen or thawed) [25]. The number of principal components was chosen by exploring the eigenvalues plot. The outliers were identified and excluded from subsequent analyzes, exploring the Hotelling's $\mathrm{T}^{2}$ - $\mathrm{Q}$ residuals plot.

\section{E. Partial Least Squares Discriminant Analysis classification}

The Partial Least Squares Discriminant Analysis (PLSDA), a pattern recognition method, was chosen to classify the spectral data of the samples [26, 27] according to the type of pasta sample (Mezze Penne and Pennette72, characterized by two different levels of saltiness) and their physical condition (i.e. frozen/thawed). Two PLS-DA models were thus developed.

To set the PLS-DA models, for the different kinds of pasta investigated conditions, the dataset of the collected spectra was randomly divided into two parts, using the Kennard-Stone algorithm [28]. The $70 \%$ of the spectral data was used to calibrate each model, while the remaining $30 \%$ was used to perform model validation. The Venetian Blinds crossvalidation algorithm was used to evaluate the optimal complexity of the model and to choose the number of latent variables (LVs). Finally, the models were tested on the validation set. The confusion matrix was considered to evaluate the classifier performance, alongside the commonly used performance metrics, calculated from it: Precision, Accuracy, Efficiency and Sensitivity and the complementary of the former, Specificity. Multi-class Precision, Accuracy and Efficiency in prediction were calculated by the harmonic mean of the class parameter.

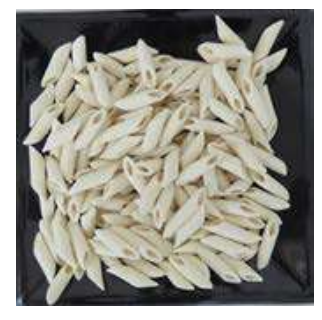

a

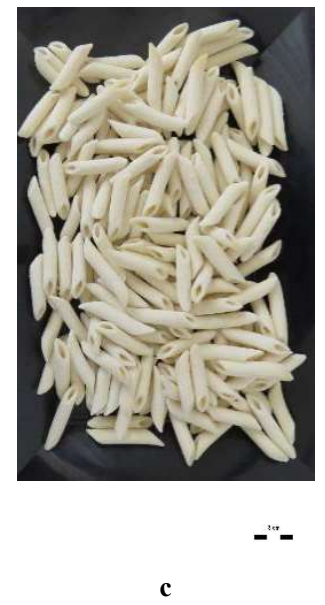

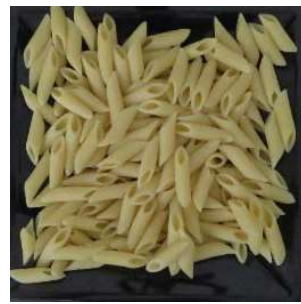

-" b

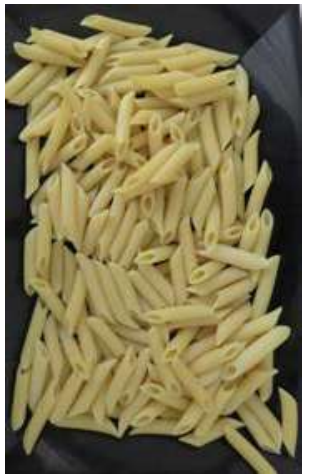

d
Fig. 1. Example of the pasta samples analyzed. Frozen (a) and thawed (b) Mezze Penne pasta and frozen (c) and thawed (d) Pennette 72 pasta. 


\section{RESULTS AND DISCUSSION}

\section{A. Exploratory analyses}

The collected raw reflectance and pre-processed spectra of the samples are reported in Fig. 2. The averaged spectra of pasta products characterized by different salting differ mostly in the visible region as can be seen in the pre-processed spectra plots (Fig. 2a and Fig. 2b). While the averaged spectra of the frozen and thawed samples mostly differ in intensities around the most of the whole spectral range considered and in spectral signature shape around 1400-1800 $\mathrm{nm}$ and 2100-2400 nm (Fig. 2c and Fig. 2d).

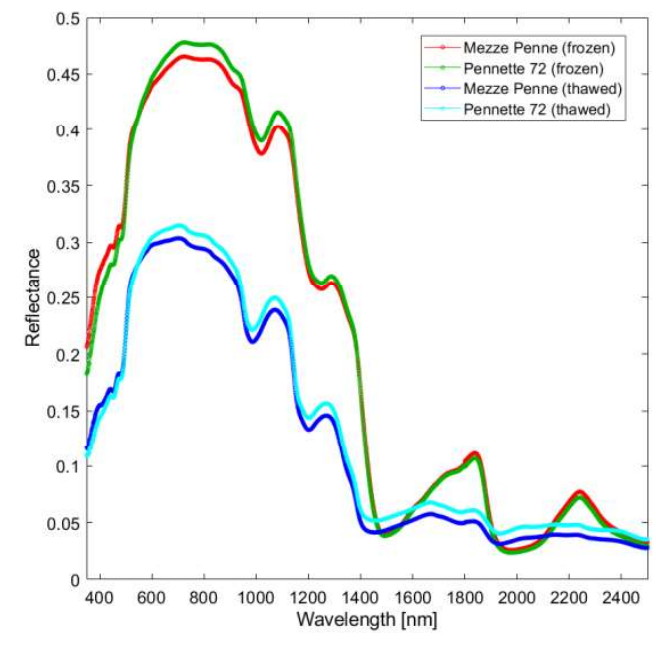

a

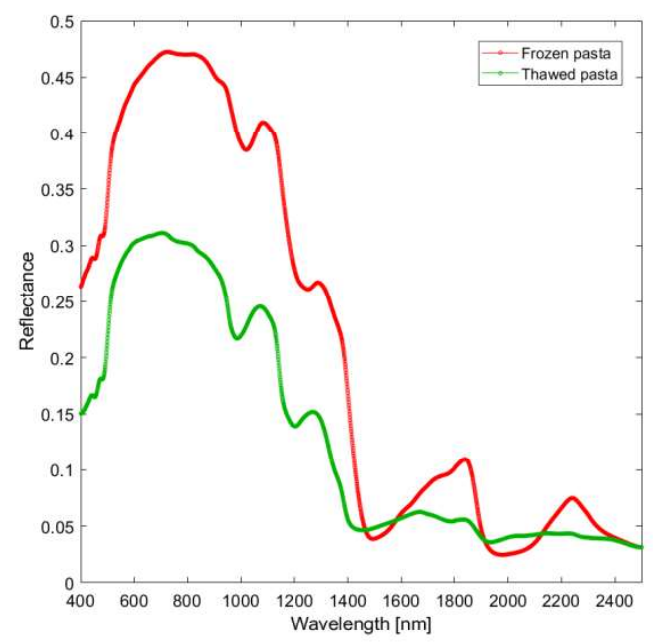

c

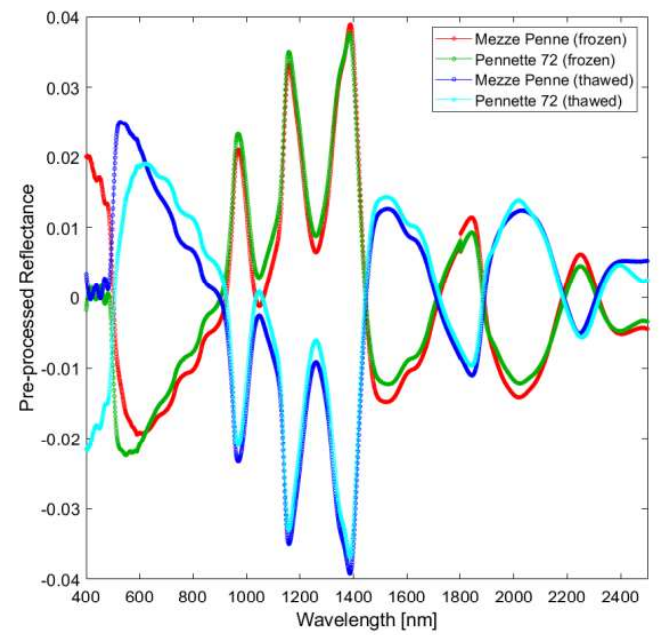

b

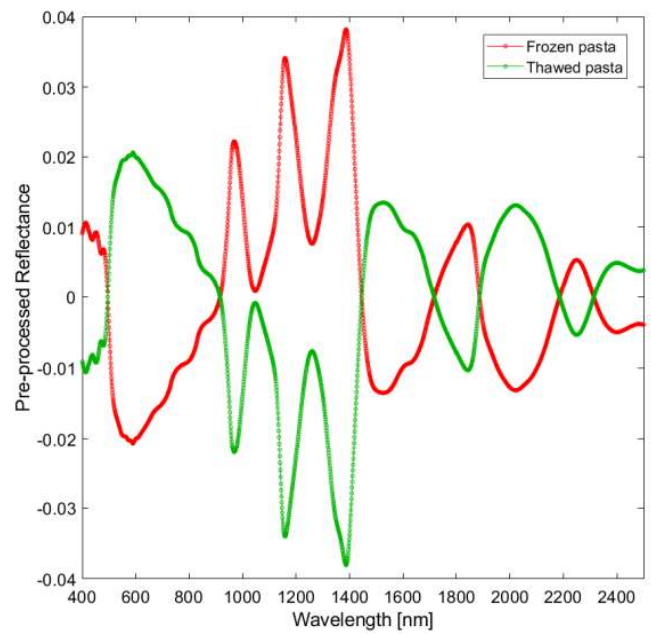

d

Fig. 2. Raw reflectance spectra (a) and preprocessed spectra averaged for pasta type and sample physical condition (b) and, raw reflectance spectra (c) and preprocessed spectra (d) averaged only for physical condition (frozen and thawed).

As can be seen in the PCA score plots of the first two principal components (PCs), the scores of the different types of pasta spectra seem to be clustering in different groups according to the second PC (resolving $8 \%$ of variance, as shown in Fig. 3a), while the defrosting process of the samples is well resolved by the first PC with an explained variance of $85 \%$ (Fig. 3b).

\section{B. Classifier performances}

The performance metrics of the PLS-DA classifier set up to discriminate the type of pasta samples (Mezze Penne and Pennette 72, characterized by two different levels of salting) and their physical condition (frozen and thawed) are reported in Table I. This four-classes model reached in prediction an Accuracy of 0.87, a Precision of 0.80 and an Efficiency of 0.84 . 
The performance metrics of the PLS-DA classifier set up to discriminate only the physical condition of the pasta samples (frozen and thawed) are reported in Table II. This two-classes classification shows very satisfactory results, reaching in prediction an Accuracy, a Precision, and an Efficiency next to the unit.

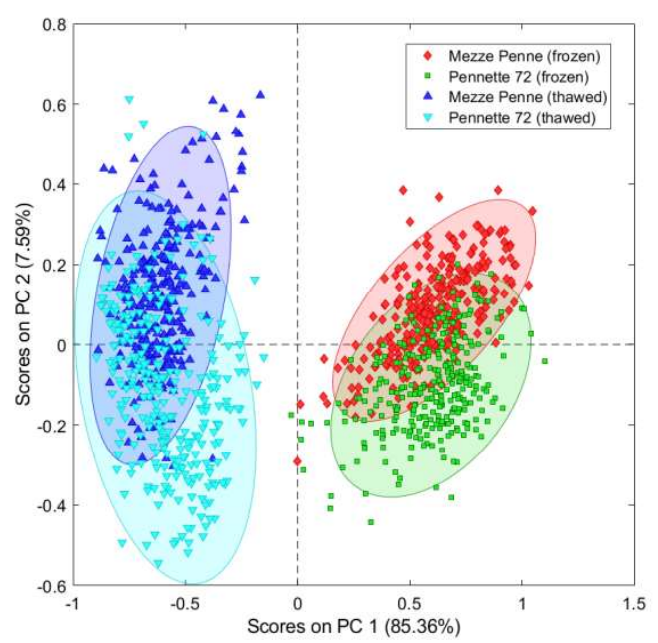

\section{CONCLUSION AND FUTURE PERSPECTIVES}

In this study, the variability of reflectance spectra in the Vis-SWIR range (350-2500 $\mathrm{nm})$ of pre-cooked pasta samples, with two levels of salting and in two different physical conditions (being frozen/thawed) was explored.

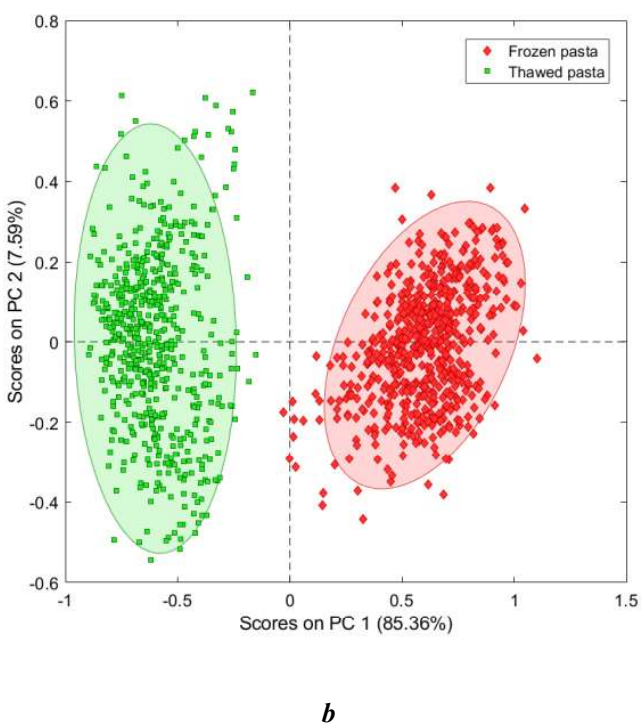

Fig. 3. PCA score plots of the decomposed spectral data according to pasta type and physical condition (a) and only according to the physical condition (b). The PC2 seems to resolve the differences between the pasta products at different saltiness level, while the PC1 is able to explain the physical condition of the products.

TABLE I. PLS-DA CLASSIFICATION PERFORMANCE METRICS TO IDENTIFY THE PHYSICAL CONDITION AND THE TYPE OF PASTA SAMPLE.

\begin{tabular}{|c|c|c|c|c|c|}
\hline $\begin{array}{l}\text { Modelled } \\
\text { class }\end{array}$ & Sensitivi & becific & recisic & ccurac & fficiency \\
\hline Mezze & & & & & \\
\hline $\begin{array}{l}\text { Penne } \\
\text { (frozen) }\end{array}$ & 0.80 & 0.93 & 0.83 & 0.89 & 0.86 \\
\hline $\begin{array}{l}\begin{array}{l}\text { Pennette72 } \\
\text { (frozen) }\end{array} \\
\text { (a) }\end{array}$ & 0.85 & 0.91 & 0.82 & 0.89 & 0.88 \\
\hline Mezze & & & & & \\
\hline $8\left[\begin{array}{l}\text { Penne } \\
\text { (thawed) }\end{array}\right.$ & 0.82 & 0.95 & 0.78 & 0.93 & 0.88 \\
\hline $\begin{array}{l}\text { Pennette72 } \\
\text { (thawed) }\end{array}$ & 0.80 & 0.96 & 0.84 & 0.93 & 0.88 \\
\hline $\begin{array}{l}\text { Mezze } \\
\text { Penne } \\
\text { (frozen) }\end{array}$ & 0.80 & 0.93 & 0.83 & 0.89 & 0.86 \\
\hline $\begin{array}{l}\text { Pennette72 } \\
\text { (frozen) }\end{array}$ & 0.84 & 0.91 & 0.81 & 0.89 & 0.88 \\
\hline \begin{tabular}{l|l} 
Mezze \\
Penne
\end{tabular} & 081 & 095 & 078 & 093 & 088 \\
\hline (thawed) & 0.01 & 0.93 & 0.10 & 0.93 & 0.88 \\
\hline$\left\{\begin{array}{l}\text { Pennette } 72 \\
\text { (thawed) }\end{array}\right.$ & 0.80 & 0.96 & 0.83 & 0.93 & 0.88 \\
\hline Mezze & & & & & \\
\hline $\begin{array}{l}\text { Penne } \\
\text { (frozen) }\end{array}$ & 0.87 & 0.96 & 0.75 & 0.95 & 0.91 \\
\hline $\begin{array}{l}\text { Pennette72 } \\
\text { (frozen) }\end{array}$ & 0.61 & 0.98 & 0.77 & 0.95 & 0.77 \\
\hline స̃. Mezze & & & & & \\
\hline$\equiv \mid \begin{array}{l}\text { Penne } \\
\text { (thawed) }\end{array}$ & 0.82 & 0.86 & 0.82 & 0.85 & 0.84 \\
\hline $\begin{array}{l}\text { Pennette } 72 \\
\text { (thawed) }\end{array}$ & 0.78 & 0.88 & 0.79 & 0.85 & 0.83 \\
\hline
\end{tabular}


Partial Least Squares - Discriminant Analysis (PLS-DA) classification models were calibrated and validated to recognize the samples according to their salting and to their physical condition, starting from their spectral signatures. Classification performances, in terms of Accuracy, Precision and Efficiency, showed promising ability in recognizing samples according to their saltiness level and to their physical condition, that is being frozen or being thawed.

Combining reflectance spectroscopy technique with a chemometric approach (i.e. PLS-DA), several goals were achieved, that is: i) the acquisition of spectral information in the Vis-SWIR range, ii) the application of pre-processing strategies able to highlight spectral differences (i.e. calibration dataset definition) and iii) the further definition of classification models, allowing to identify pasta characteristics and status, both issues not easy to assess with a classical approach, due to the very similar physical-chemical characteristics of the products.

The application of these techniques, if fully implemented, can be functional both at industrial-scale production, to develop, implement and set up fast, robust and reliable product quality control applications (i.e. salting and defrosting process evaluation), and also to products status in the distribution chain (i.e. cold chain quality).

Future works will be addressed to strengthen the logic presented in this paper in a full industrial-scale application perspective.

TABLE I. PLS-DA CLASSIFICATION PERFORMANCE METRICS TO IDENTIFY THE PHYSICAL CONDITION OF THE PASTA SAMPLE.

\begin{tabular}{|c|c|c|c|c|c|c|}
\hline & $\begin{array}{c}\text { Modelled } \\
\text { class }\end{array}$ & & & $c$ & & \\
\hline .5 & $\begin{array}{c}\text { Frozen } \\
\text { pasta }\end{array}$ & 1 & 1 & 1 & 1 & 1 \\
\hline తే & $\begin{array}{l}\text { Thawed } \\
\text { pasta }\end{array}$ & 1 & 1 & 1 & 1 & 1 \\
\hline 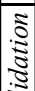 & $\begin{array}{c}\text { Frozen } \\
\text { pasta }\end{array}$ & 1 & 1 & 1 & 1 & 1 \\
\hline $\begin{array}{l}1 \\
\vdots \\
\vdots \\
0 \\
0\end{array}$ & $\begin{array}{c}\text { Thawed } \\
\text { pasta }\end{array}$ & 1 & 1 & 1 & 1 & 1 \\
\hline. & $\begin{array}{c}\text { Frozen } \\
\text { pasta }\end{array}$ & 1 & 1 & 1 & 1 & 1 \\
\hline$=$ & $\begin{array}{l}\text { Thawed } \\
\text { pasta }\end{array}$ & 1 & 1 & 1 & 1 & 1 \\
\hline
\end{tabular}

\section{ACKNOWLEDGMENT}

The authors wish to thank the company Gelit SpA to have provided the samples object of the study and for the profitable analysis and discussions of the results.

\section{REFERENCES}

[1] E. Tamburini, M. Battaglia, L. Suma, and M. M.G., "La tecnica NIR applicata al controllo qualità per la produzione di pasta di semola, pasta all'uovo," 2016 [Atti del congresso NIR ITALIA 2016].

[2] S. Zardetto, "Potential applications of near infrared spectroscopy for evaluating thermal treatments of fresh egg pasta," Food Control, 2005, pp. 249-256.

[3] F. Groß, R. Benning, U. Bindrich, K. Franke and V. Heinz, "Optical online measurement technique used for process control of the drying step during pasta production," Procedia Food Science, 1, pp. 1301 1308, 2011 [11th International Congress on Engineering and Food (ICEF11), 2011].

[4] M. Bevilacqua, R. Bucci, S. Materazzi, and F. Marini, "Application of near infrared (NIR) spectroscopy coupled to chemometrics for dried egg-pasta characterization and egg content quantification," Food Chemistry, 140, 2013, pp. 726-734.

[5] H. Masoumi, S. M. Safavi, and Z. Khani, "Identification and Classification of Plastic Resins using Near Infrared Reflectance
Spectroscopy," International Journal of Mechanical, Aerospace, Industrial, Mechatronic and Manufacturing Engineering, 6(5), 2012, pp. 877-884

[6] G. Bonifazi, R. Gasbarrone, R. Palmieri, and S. Serranti, "Near infrared hyperspectral imaging-based approach for end-of-life flat monitors recycling," at - Automatisierungstechnik, 68 (4), 2020, pp. 265-276.

[7] G. Agresti, G. Bonifazi, G. Capobianco, L. Lanteri, C. Pelosi, S. Serranti, and A. Veneri, "Hyperspectral imaging as powerful technique for evaluating the stability of Tattoo Wall@," Microchemical Journal, 104866, 2020.

[8] G. Bonifazi, G. Capobianco, C. Pelosi, and S. Serranti,. Hyperspectral imaging as powerful technique for investigating the stability of painting samples. Journal of Imaging, 5(1), 8, 2019.

[9] C. A. Teixeira dos Santos, M. Lopo, R. N.M.J. Páscoa, and J. A. Lopes, "A Review on the Applications of Portable Near-Infrared Spectrometers in the Agro-Food Industry," Applied Spectroscopy, 67 (11), 2013, pp. 1215-1233.

[10] C. Kumaravelu, and A. Gopal, "A Review on the applications of NearInfrared Spectrometer and Chemometrics for the Agro-Food Processing Industries," Proc. 2015 IEEE International Conference on Technological Innovations in ICT for Agriculture and Rural Development, 2015.

[11] S. Serranti, G. Bonifazi, R. Gasbarrone, "Olive fruit ripening evaluation and quality assessment by hyperspectral sensing devices, "Proc. SPIE 
10665, Sensing for Agriculture and Food Quality and Safety X, 106650R (15 May 2018).

[12] S. Serranti, G. Bonifazi, R. Gasbarrone, "Kiwifruits ripening assessment by portable hyperspectral devices", Proc. SPIE 10665, Sensing for Agriculture and Food Quality and Safety X, 106650S (15 May 2018).

[13] G. Bonifazi, R. Gasbarrone, and S. Serranti, "Dried red chili peppers pungency assessment by visible and near infrared spectroscopy, " Proc. SPIE 10986, Algorithms, Technologies, and Applications for Multispectral and Hyperspectral Imagery XXV, 109861S (14 May 2019);

[14] S. Tsuchikawa, and H. Kobori, "A review of recent application of near infrared spectroscopy to wood science and technology," J Wood Sci, 61, 2015, pp. 213-220.

[15] Y. Roggo, P. Chalus, L. Maurer, C. Lema-Martinez, A. Edmond, and N. Jent, "A review of near infrared spectroscopy and chemometrics in pharmaceutical technologies," Journal of Pharmaceutical and Biomedical Analysis, 44, 2007, pp. 683-700.

[16] M.S. Larrechi, and M.P. Callao, "Strategy for introducing NIR spectroscopy and multivariate calibration techniques in industry," Trends in Analytical Chemistry, 22 (10), 2003.

[17] G. Bonifazi, A. Cardillo, A. Currà, R. Gasbarrone, S. Serranti, "Near infrared spectroscopy as a tool for in vivo analysis of human muscles," Proc. SPIE 10662, Smart Biomedical and Physiological Sensor Technology XV, 1066200 (14 May 2018).

[18] R. Gasbarrone, A. Currà, A. Cardillo, G. Bonifazi, S. Serranti, "Near infrared spectroscopy of human muscles,"Proc. SPIE 10489, Optical Biopsy XVI: Toward Real-Time Spectroscopic Imaging and Diagnosis, 1048914 (21 February 2018).

[19] A. Currà, R. Gasbarrone, A. Cardillo, C. Trompetto, F. Fattapposta, F. Pierelli, P. Missori, G. Bonifazi, and S. Serranti, "Near infrared spectroscopy as a tool for in vivo analysis of human muscles," Scientific Reports 9, 8623, 2019.

[20] C. Pasquini, "Near Infrared Spectroscopy: Fundamentals, Practical Aspects and Analytical Applications," J. Braz. Chem. Soc., 14 (2), 2003, pp. $198-219$.

[21] ASD Inc., "FieldSpec ${ }^{\circledR} 4$ User Manual”, ASD Document 600979, Rev. D. 2015

[22] B. M. Wise, N. B. Gallagher, R. Bro, J. M. Shaver, W. Windig, and R. S. Koch, "PLS_Toolbox 4.2 Reference Manual for use with

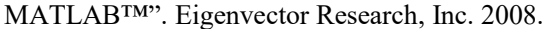

[23] M. Danner, M. Locherer, T. Hank, and K. Richter. Spectral Sampling with the ASD FieldSpec 4 -Theory, Measurement, Problems, Interpretation. EnMAP Field Guides Technical Report, GFZ Data Services. (2015)

[24] Å. Rinnan, F. van den Berg, and S. B. Engelsen, "Review of the most common pre-processing techniques for near-infrared spectra," Trends in Analytical Chemistry, 28 (10), 2009, pp. 1201-1222.

[25] S. Wold, K. Esbensen, and P. Geladi, "Principal component analysis," Chemometrics and Intelligent Laboratory Systems, 2(1-3), 1987, pp. 3752.

[26] M. Barker, and W. Rayens, Partial least squares for discrimination. Journal of Chemometrics, 17, 2003 pp. 166-173.

[27] D. Ballabio, and V. Consonni, "Classification tools in chemistry. Part 1: linear models. PLS-DA," Analytical Methods, 5, 2013, pp. 3790-3798.

[28] R. Kennard, and L. Stone, "Computer Aided Design of Experiments," Technometrics, 11(1), 1969, pp. 137-148. 